\title{
Gamma-ray line emission from galaxy clusters ${ }^{\star}$
}

\author{
A. F. Iyudin ${ }^{1}$, H. Böhringer ${ }^{1}$, V. Dogiel ${ }^{2}$, and G. Morfill ${ }^{1}$ \\ 1 Max-Planck-Institut für extraterrestrische Physik, Postfach 1312, 85741 Garching, Germany \\ 2 P.N. Lebedev Physical Institute, RAS, 117924 Moscow, Russia
}

Received 31 March 2003 / Accepted 23 September 2003

\begin{abstract}
We report first results of a study dedicated to a search for $\gamma$-ray line emission from rich galaxy clusters, finding traces of emission from the region of Virgo and Coma near the Northern Galactic Pole (NGP). The study is based on the 6 years of COMPTEL data collected during the extended observational programme of the Compton Gamma Ray Observatory (CGRO) monitoring of the Virgo and 3C273 region. We point out the advantages of performing this study using an energy range that contains the strongest excitation $\gamma$-ray lines of ${ }^{16} \mathrm{O}$ at $6.13,6.9$ and $7.1 \mathrm{MeV}$, as well as lines at $6.175 \mathrm{MeV}\left({ }^{15} \mathrm{O}^{*}\right)$ and $6.322 \mathrm{MeV}\left({ }^{15} \mathrm{~N}^{*}\right)$ of the spallation products of the cosmic ray interactions with ${ }^{16} \mathrm{O}$. We discuss a generation mechanism for $\gamma$-ray line emission in merging clusters via excitation of the abundant element(s) of the intracluster matter at the merger shock by the subrelativistic protons accelerated to energies of $\sim 10 \mathrm{MeV}$ to $\sim 100 \mathrm{MeV} / \mathrm{n}$ in the merger process.
\end{abstract}

Key words. galaxy clusters: general - galaxies: clusters: individual: Virgo - gamma-rays: observation

\section{Introduction}

Major galaxy cluster mergers are the most energetic events in the Universe since the Big Bang (Sarazin 2002). In such mergers, the subclusters collide at velocities of up to $\sim 2000 \mathrm{~km} \mathrm{~s}^{-1}$, leading to hydrodynamical shocks that dissipate energies up to $\sim 3 \times 10^{63}$ ergs. The merger shock velocities are similar to those in supernova remnants (SNRs) in our Galaxy, and it is reasonable to expect them to produce nonthermal electrons and ions. Cosmic Rays (CRs) in clusters of galaxies, if present, could provide a source of substantial internal energy of the intracluster medium (ICM). CRs together with the ICM magnetic field whose configuration is strongly influenced by ongoing or past mergers, will build up a non-thermal pressure in the ICM that makes the assumption of the purely thermal hydrostatic equilibrium mass distrubution in the cluster imprecise. Additionally, the non-symmetric magnetic field configuration will impact on the cluster cooling. The importance of CRs in cooling of clusters of galaxies was early realised and analysed in work by Böhringer \& Morfill (1988) and recently by Loeb (2000). Unfortunately, until now no definite proof for the presence of a non-thermal component of nucleonic cosmic rays in galaxy clusters was found.

Previous attempts to detect possible signatures of galaxy clusters in the $\gamma$-ray domain based on the analysis of EGRET

Send offprint requests to: A. F. Iyudin, e-mail: ani@mpe.mpg. de

* The COMPTEL project is supported by the BMBF through DLR grant 50 QV 90968 . AFI acknowledges financial support from the BMBF through the DLR grant 50 OR 0002. data were not successful (Shreekumar et al. 1996; Reimer et al. 2003). Recently associations of rich clusters with a few unidentified high-latitude EGRET sources were proposed, but in all such cases an association was claimed with a rather low significance level $(<3 \sigma)$ (Collafranchesco 2002; Sharf \& Mukherdjee 2002; Kawasaki \& Totani 2002).

No investigation of galaxy clusters as a class of objects to be associated with MeV $\gamma$-ray emission has been carried out yet. We try to fill this gap by starting a study of the possible association of $\mathrm{MeV} \gamma$-ray emission with the clusters in the region near the Northern Galactic Pole (NGP) based on the COMPTEL survey data. The NGP region was well observed by COMPTEL during monitoring of two quasars 3C273 and $3 \mathrm{C} 279$, and observations of the Virgo region, with an exposure of $\sim 6.1 \times 10^{6} \mathrm{~s}$ for Coma, and $\sim 6.9 \times 10^{6} \mathrm{~s}$ for Virgo.

The non-thermal component of subrelativistic ions in clusters can be detected by tracing their interaction with the ICM that leads to the production of de-excitation $\gamma$-ray line emission. Our study has established an association of the most significant $\gamma$-ray line emission excesses $(\geq 5 \sigma)$ with the rich clusters Virgo and Coma. These results are discussed below.

The paper is organized as follows. In Sect. 2 we discuss the instrument characteristics and the basis for using excitation lines of ${ }^{16} \mathrm{O}$ for the galaxy clusters study. In Sect. 3 we describe methods used in this work for the imaging and spectral analysis of COMPTEL data. Section 4 presents results and in Sect. 5 we discuss results, processes of the $\gamma$-ray line generation in merging galaxy clusters, and finally give a summary. 


\section{Instrument and data}

COMPTEL aboard the Compton Gamma-Ray Observatory (CGRO) was the first $\gamma$-ray imaging telescope capable to perform an all sky survey at $\mathrm{MeV}$ energies. In the $\gamma$-ray sky many types of objects were already detected by COMPTEL. Among them are active galactic nuclei (AGN), pulsars, X-ray binaries and SNRs (Schönfelder et al. 2000), but a new class of $\gamma$-ray emitting objects may be expected as well in particular in the relatively unexplored field of the $\mathrm{MeV} \gamma$-ray astronomy. COMPTEL operates in the 0.75 to $30.0 \mathrm{MeV}$ energy range with a field-of-view of about 1 steradian, a source location accuracy of $\sim 1^{\circ}$, and an energy resolution of $5-10 \%$ FWHM. A detailed description of the instrument, its calibration, and the COMPTEL data analysis in the 3-dimensional data space is given by Schönfelder et al. (1993). Here we present a study of $\gamma$-ray emission from galaxy clusters performed in the energy range 5.6-7.6 MeV, that includes the strongest ${ }^{16} \mathrm{O}$ lines at $6.13 \mathrm{MeV}, 6.917 \mathrm{MeV}$ and $7.117 \mathrm{MeV}$, as well as the first escape peak of the $6.13 \mathrm{MeV}$ line. These lines are expected to be produced in interactions of the subrelativistic CRs with the ICM ions, like in the inelastic nuclear reaction of the type ${ }^{16} \mathrm{O}\left(\mathrm{p}, \mathrm{p}^{\prime}\right)^{16} \mathrm{O}^{*}$. The choice of this energy interval follows from the emission spectrum expected to be produced by subrelativistic protons and nuclei accelerated by shock waves resulting from the merging process, and interacting with the gas and dust of ICM. Assuming usual relative abundances of the ICM elements, we have simulated an expected $\gamma$-ray spectrum from such interactions. In performing simulations we have followed the approach developped by Ramaty et al. (1996) for a thick target case with the use of an algorithm described by Kozlovsky et al. (2002), and of the accelerated particle source spectrum of the form (Ramaty et al. 1996)

$$
\frac{\mathrm{d} N_{i}}{\mathrm{~d} t}\left(E_{n}\right) \propto E_{n}^{-\alpha} \mathrm{e}^{-E_{n} / E_{0}}
$$

The simulated spectrum contains many lines near $\sim 1 \mathrm{MeV}$, that will blend in the spectrum of the telescope with moderate energy resolution. Contrary to the $\sim 1 \mathrm{MeV}$ region, regions around $4.4 \mathrm{MeV}$ and $6.13 \mathrm{MeV}$ lines are relatively free from other lines. These lines at $4.4 \mathrm{MeV}$ of ${ }^{12} \mathrm{C}^{*}$ and at $6.13 \mathrm{MeV}$ of ${ }^{16} \mathrm{O}^{*}$ are separated well enough to potentially simplify the analysis of the line broadening, line shifts etc., for a telescope with a moderate energy resolution like COMPTEL, which has $\delta E / E=5 \%$ at $\sim 6 \mathrm{MeV}$. Figure 1 shows the simulated spectrum in the region of ${ }^{16} \mathrm{O}$ lines after convolution with the response function of COMPTEL. Note, that the majority of the $6.13 \mathrm{MeV}$ line photons will be absorbed in the D2 modules of COMPTEL via $\mathrm{e}^{-} \mathrm{e}^{+}$pair-production, which cause an appearance of a shoulder in the convolved spectrum of Fig. 1 at $\sim 5.6 \mathrm{MeV}$. This shoulder is produced by the single escape photons of the $6.13 \mathrm{MeV}$ line. In our study we prefer to use ${ }^{16} \mathrm{O}$ lines as a probe of the non-thermal component in clusters and not the $4.4 \mathrm{MeV}$ line of ${ }^{12} \mathrm{C}$. The reasons are as follows: (1) - a large part of the $4.4 \mathrm{MeV}$ line flux can be produced by the spallation reaction on ${ }^{16} \mathrm{O}$; and (2) - the $4.4 \mathrm{MeV}$ line is rather close to the intense and strongly variable instrumental background line at $\sim 3.8-3.9 \mathrm{MeV}$ (see Sect. 3.2).

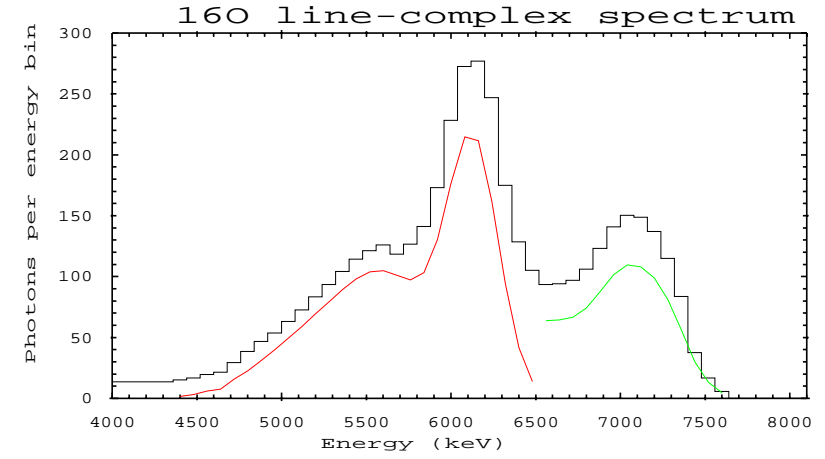

Fig. 1. Model spectrum of the cluster ${ }^{16} \mathrm{O} \gamma$-ray lines excess convolved with the energy response matrix of COMPTEL. The most intense line corresponds to the $6.13 \mathrm{MeV}$ line of ${ }^{16} \mathrm{O}$, which has also a low energy shoulder that is due to the $6.13 \mathrm{MeV}$ line 1st escape peak. The broad feature at $\sim 7 \mathrm{MeV}$ is a blend of two ${ }^{16} \mathrm{O}$ lines at $6.97 \mathrm{MeV}$ and $7.17 \mathrm{MeV}$.

The imaging data space of the Compton telescope is spanned by three coordinates, with two angles $\chi$ and $\psi$ of the scattered photon direction (the scatter direction) and the scatter angle $\bar{\varphi}$. The scatter angle $\bar{\varphi}$ is calculated from measured energy losses in two detectors using the Compton-scatter formula, while angles $\chi$ and $\psi$ are determined by the interaction locations of the $\gamma$-ray photon in the D1 and D2-detectors (Schönfelder et al. 1993). The 3D data space binning is $1^{\circ} \times$ $1^{\circ} \times 2^{\circ}$ in $\chi, \psi, \bar{\varphi}$. In the COMPTEL imaging analysis $\gamma$-ray albedo photons from the Earth's atmosphere are rejected by determining for each event the minimal angular distance $(\zeta)$ between its possible origins in the sky and the Earth horizon (Schönfelder et al. 1993).

In this work we have used the standard selection set of COMPTEL, with an addition of a more stringent constraint on the minimal angle $\zeta\left(\zeta \geq 10^{\circ}\right)$ for photons used in our imaging and spectral analysis (see details in Schönfelder et al. 1993). We have chosen this event selection set after comparing selection sets that differ in the minimum Earth-horizon event angle $\left(\zeta \geq 0^{\circ}\right.$, or $\geq 10^{\circ}$ ), and in the time-of-flight (ToF) window used, like: standard 115-130, or, alternatively, a ToF window at 112-127 and PSD window of 67-87, i.e., the so-called selection set TOFT43. In this comparison four combinations of two selection datasets (standard MPE006 and TOFT43) and of two minimum Earth-horizon event angle restrictions were used to produce four data sets for the 5.6-7.6 MeV energy interval. We present results of the comparative imaging analysis of the NGP region data with four different selection sets in Table 1. This region (NGP) was observed quite regularly, while monitoring activity of two $\gamma$-ray bright QSOs, 3C273 and 3C279. Overall $\sim 40$ observations covering NGP region were used in this study, with the total exposure reaching $\sim 7.0 \times 10^{6} \mathrm{~s}$ at the position of $3 \mathrm{C} 273$. Judging by the values of error counts recovered from the positions of $3 \mathrm{C} 273$, 3C279, Coma cluster, Virgo cluster and Abell 1465, as well as by the integral value of the likelihood ratio improvement over the value derived for a flat flux distribution in the NGP region, we finally selected the combination of MPE006 (standard selection data-set) and of Earth-horizon event angle $\zeta \geq 10^{\circ}$. 
Table 1. Number of error counts in the energy interval $5.6 \mathrm{MeV} \leq E_{\gamma} \leq 7.6 \mathrm{MeV}$ at positions of the excesses and the total maximum-likelihood ratio $(-2 \ln L)$ relative to a flat background model fitting.

\begin{tabular}{rcccc}
\hline \hline Object & MPE006, $\zeta \geq 10^{\circ}$ & TOFT43, $\zeta \geq 10^{\circ}$ & MPE006, $\zeta \geq 0^{\circ}$ & TOFT43, $\zeta \geq 0^{\circ}$ \\
\hline 3C 273 & 343 & 360 & 376 & 405 \\
3C 279 & 325 & 345 & 356 & 387 \\
Virgo & 341 & 358 & 374 & 402 \\
Coma & 273 & 290 & 302 & 324 \\
A1465 & 252 & 267 & 283 & 303 \\
\hline$-2 \ln L^{a}$ & 79.7 & 62.1 & 66.0 & 69.5 \\
\hline
\end{tabular}

a) Value of the maximum-likelihood ratio $(-2 \ln L)$ relative to the flat background model fitting.

\section{Imaging and spectral analysis of the NGP region data}

\subsection{Imaging analysis}

For the imaging analysis we have used a version of the maximum-likelihood method (SRCFIX), that evolved from the work on diffuse emission modeling in the COMPTEL data space (van Dijk 1996). In this imaging program an internal background model is generated using a filtering technique for each observation, which takes into account the variation of the instrumental background with time. This technique is based on the fact that the $(\chi, \psi)$ structure of the data cube is well described to first order by the known geometry characteristics, with deviations being largely independent of $\bar{\varphi}$ (Bloemen et al. 1994). Note that COMPTEL's instrumental background compromises $\sim 98 \%$ of the total background in the $1-3 \mathrm{MeV}$ energy interval, but decreases significantly above an energy of $\sim 4.3 \mathrm{MeV}$. An iterative approach (background modeling and sky-model fitting being performed simultaneously) was added to this method (Bloemen et al. 1999, 2000). In this approach all observations are again handled separately, in order to account for changes in the instrumental background during the mission.

In the following the display of the maximum-likelihood ratios over the field of view is refered to as the maximumlikelihood (ML) map. Such maps allow the determination of the excess significance, flux, error region, etc. Our imaging analysis has established a positive correlation between the significant $\gamma$-ray line emission excesses $(\geq 3 \sigma)$ and the nearby rich clusters. In total we have detected 10 excesses in the NGP region (Fig. 2), with two most significant excesses that are positionally coincident with the rich clusters Virgo and Coma.

Figure 2 presents a likelihood ratio contour map of the NGP $\gamma$-ray line excesses that exceed $3 \sigma$ detection significance for a 1 d.o.f. search. Figures 3 and 4 show maps in adjacent energy bands of 4.3-5.6 MeV and 7.6-10.0 MeV where no counterpart can be found for the ${ }^{16} \mathrm{O}$ line emission excesses 1 , $2,3,5,6,7$ and 8 . The same is true if one compares positions of EGRET unidentified (UID) sources in the region from Hartman et al. (1999), with that of the line excesses. The nearest source to our excess 1 is EGRET UID 3EG J1323+2200 $\sim 4^{\circ}$ to the south-east and the nearest source to excess 2 is EGRET UID 3EG J1236+0457 about $5^{\circ}$ to the south of the line excess. Apart from the expected overlaps with two strong $\gamma$-ray sources $3 \mathrm{C} 273$ and $3 \mathrm{C} 279$ no spatial correlation of line

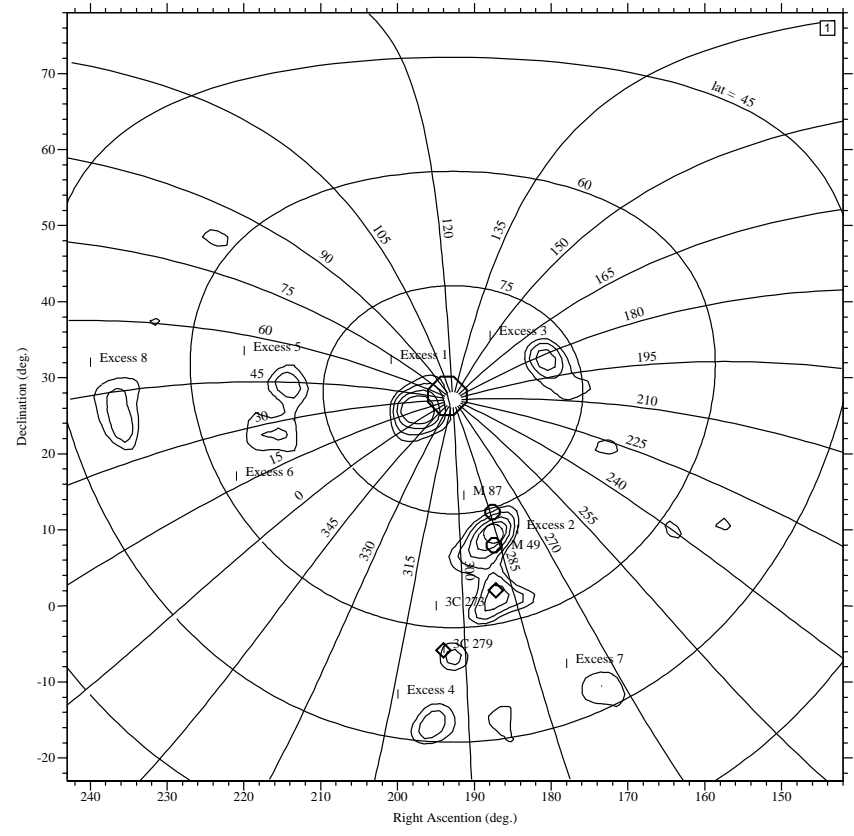

Fig. 2. A map of the North Galactic Pole region in equatorial coordinates (J2000.0) produced by the likelihood method. Contour lines are shown at a likelihood ratio value of 9, 12, 16 and 20. Small circles show the positions of M 87 and of M 49, and lozenges mark the positions of $3 \mathrm{C} 273$ and of $3 \mathrm{C} 279$. A grid of galactic coordinates is overlayed. Positions of the ${ }^{16} \mathrm{O}$ line emission excesses possibly related to galaxy clusters are marked by numbers from Excess 1 to Excess 8 .

excesses with EGRET sources (Hartman et al. 1999) can be claimed in the NGP region. The same holds for the only UID COMPTEL source GRO J1040-48 (Iyudin et al. 1996) in the region.

\subsection{Spectral analysis}

In the present study gamma-quanta for spectral analysis were spatially selected with a scatter direction and a scatter angle compatible with circular regions of $2^{\circ}$ radii centered at the position of the excesses detected in the ML-maps. This approach was described by Iyudin et al. (1994), and was followed through the later studies of $\gamma$-ray lines from different point sources (Iyudin et al. 1995, 1998). Background spectra were generated using high-latitude NGP region observations 


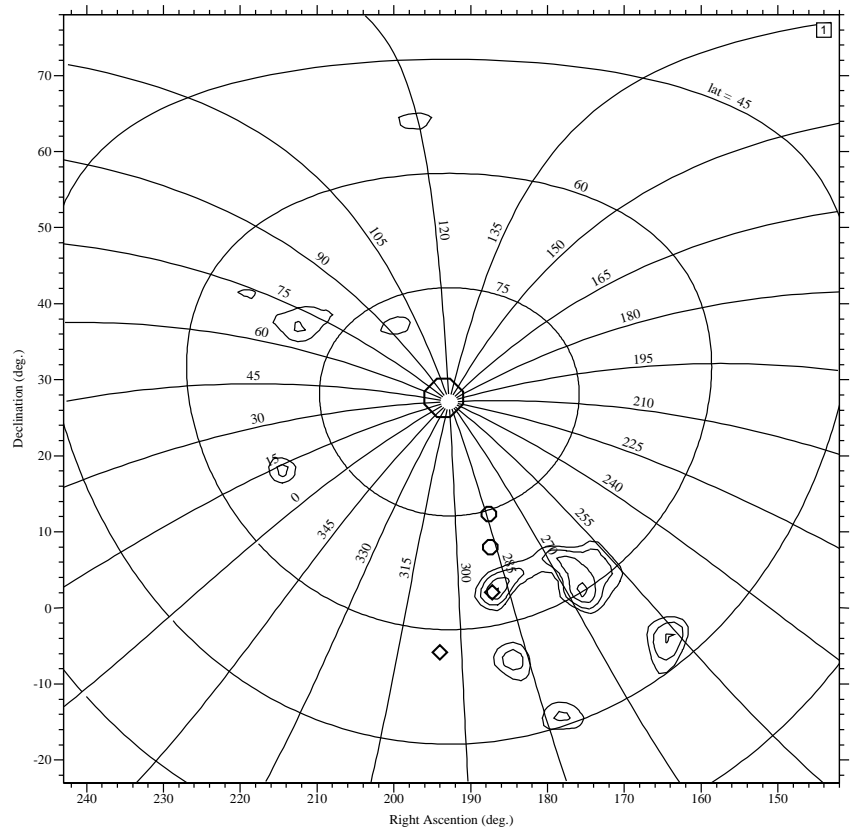

Fig. 3. The same as in Fig. 2 but for $4.3 \mathrm{MeV} \leq E_{\gamma} \leq 5.6 \mathrm{MeV}$.

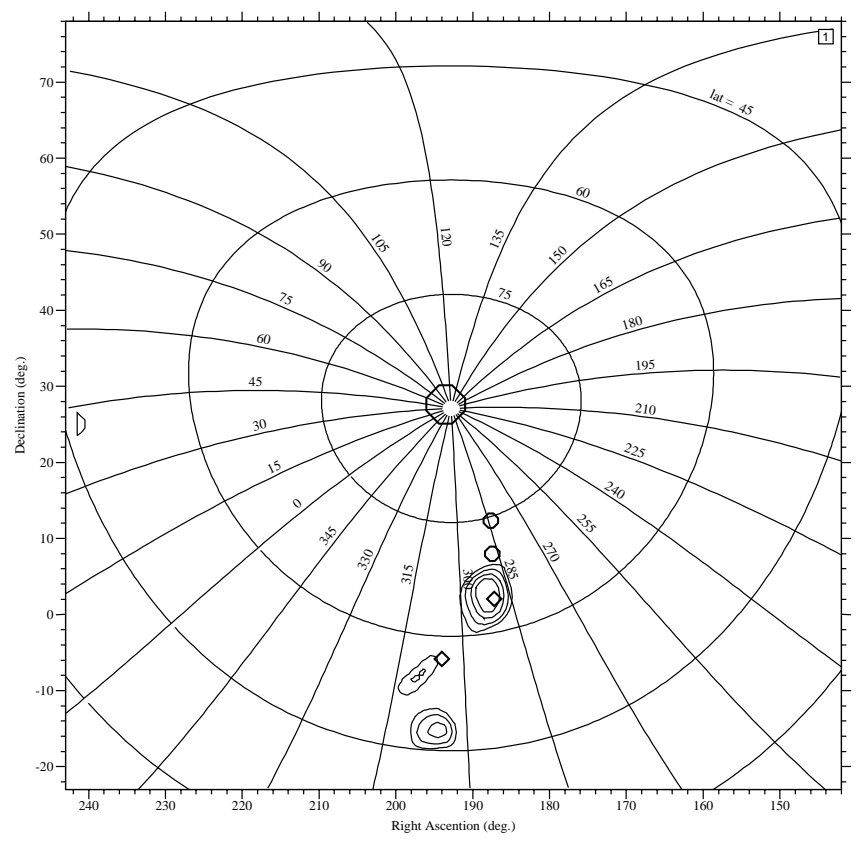

Fig. 4. The same as in Fig. 2 but for $7.6 \mathrm{MeV} \leq E_{\gamma} \leq 10 \mathrm{MeV}$.

applying identical selections and avoiding regions likely contaminated by the emission from Loop I and Loop IV.

The COMPTEL standard background model extensively used in the point-like excesses studies (Iyudin et al. 1994, 1995, 1998), usually constructed using gamma-quanta collected from the circle with large $\left(10^{\circ}\right)$ radii centered on the line excess position, is not applicable to extended emission regions, like merger regions in nearby clusters (see simulations by Ritchie \& Thomas 2001, and Sect. 4). Therefore, in this study we have used an NGP background model, normalised to the total number of counts in the source spectrum. An energy binning of $80 \mathrm{keV}$ was used throughout this study. Figure 5 shows the

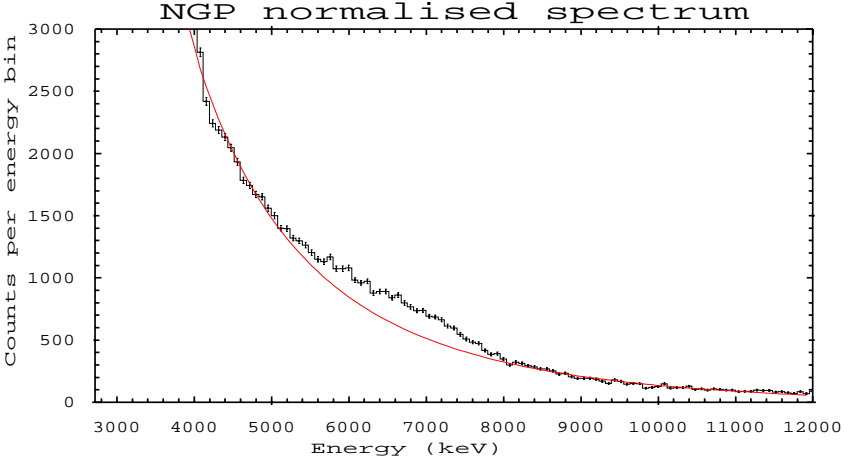

Fig. 5. Total spectrum of the sum of 94 different positions within $\sim 40$ observations of the NGP region excluding regions around supernova remnants (SNRs) of Loop I and Loop IV with boundaries given by radio measurements. The spectrum was normalised to the total number of counts of the similar Coma spectrum. The solid line gives a fit to the assumed smooth background.

background spectrum constructed from 94 pointings in the field of view of $\sim 40$ different observations of the NGP region. The spectrum of Fig. 5 normalised to the total number of counts in the raw Coma spectrum clearly contains an excess above the smooth background at energies of 5.0 to $8.0 \mathrm{MeV}$. What is the nature of this excess?

The largest part of this excess is due to the instrumental background blended lines at energies above $\sim 4.3 \mathrm{MeV}$, i.e., a spontaneous background emission that was discussed by Weidenspointner (1999). This background is produced by secondary neutrons interacting with the telescope material. These secondary neutrons, in turn, are produced by CR and radiation belt protons interacting with the instrument (and satellite) construction material. In addition to the strong instrumental background, one expects to have a small contribution of the $\gamma$-ray line emission in the same energy band from the high redshift galaxy clusters (sky background emission), and foreground emission from the Local Bubble, Loop I and Loop IV. The former was studied in X-rays as extended O VII line emission from the galaxy clusters baryonic component (Kaastra et al. 2003), and the latter is supported by the results of the local O VII and O VI studies, performed with FUSE in UV, and by Chandra and XMM-Newton in X-rays (Nicastro et al. 2002; Fang et al. 2002; Paerels et al. 2002; Savage et al. 2003; Zsargo et al. 2003), including sight lines toward bright QSOs, like PG 0953+415, PKS 2155-304, MKN 421, H1821+643 and $3 \mathrm{C} 273$.

Our study of this high-energy background component (Fig. 5) does confirm that its strength is independent of the mission-time, a result that is consistent with earlier studies (Weidenspointner 1999). Figure 6 illustrates this independence. An instrumental background degrades the sensitivity of the instrument. But quite reassuringly the signal $(S)$ to noise $(N)$ ratio at energies of ${ }^{16} \mathrm{O}$ excitation lines has a much more favourable value of $S / N \approx 0.05$ (see Figs. 5 and 7 ), compared to that of the $\sim 1 \mathrm{MeV}$ region, where COMPTEL has $S / N \approx$ 0.02 .

Let us expand on the reason for not using the $4.4 \mathrm{MeV}$ excitation line for this study. Usually, to derive the residual 


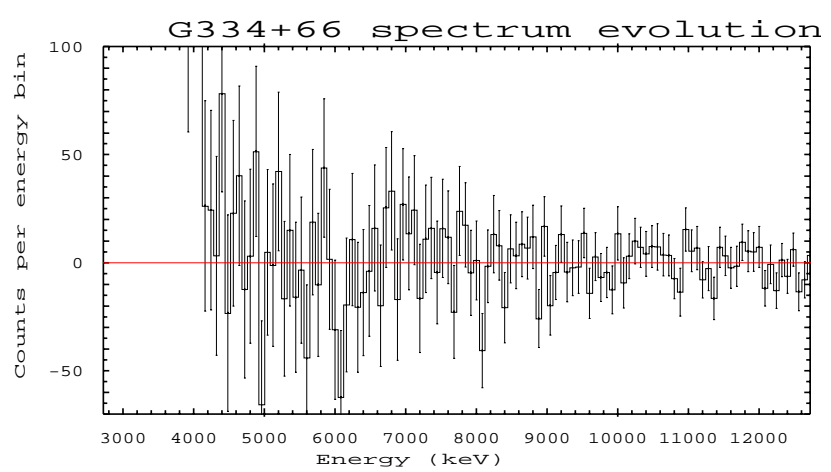

Fig. 6. Difference of two spectra at $E_{\gamma} \geq 4.0 \mathrm{MeV}$ taken from the same point on the sky with galactic coordinates $l=334^{\circ}, b=+66^{\circ}$, but for two different time intervals: first covering time from TJD 8422.5654 to TJD 9285.4181, and second covering time from TJD 9699.428 to TJD 10434.433. The time between two measuments is $\sim 3$ yrs. The solid line follows zero difference between spectra.

spectra, the "background" spectrum has to be subtracted from the "source" spectrum after normalisation to the integral number of counts at some chosen energy interval. The difference in time intervals used for the spectra accumulation leads to the different strength of the time dependent instrumental background line at 3.8-3.9 MeV in the raw spectra. After subtraction of the NGP background, very strong fluctuations are produced near the instrumental background line at $\sim 3.8 \mathrm{MeV}$, that affect the residual spectrum of COMPTEL up to $\sim 5.0 \mathrm{MeV}$. Actually, this is a known COMPTEL specific instrumental background effect that was studied in relation to the Orion nebula line emission (Bloemen et al. 1999).

\section{Results}

\subsection{Excess 1}

Excess 1 (Fig. 2) was detected by COMPTEL with $\sim 5.2 \sigma$ significance for the 1 d.o.f. search. Its likelihood-ratio peak is shifted relative to the Abell 1656 (Coma) position by $\sim 3^{\circ}$. The $2 \sigma$ error region of excess 1 contains 8 Zwicky clusters, Abell 1665 and 4 QSOs. Additional 6 QSOs have positions quite near the $2 \sigma$ error box boundary.

We can use positions of the most intense ${ }^{16} \mathrm{O}$ line at $6.13 \mathrm{MeV}$ (in the rest mass system) and of its 1st escape peak, in the spectrum of the Coma $\gamma$-ray line excess (Fig. 7), to derive the redshift of the line emitting system. The derived redshift of $z_{\text {excess } 1}=0.026 \pm 0.008$ is consistent with the Coma cluster redshift of $z_{\text {Coma }}=0.0232$. If the merging process is at a rather early stage, the merger region with the $\gamma$-ray emission can be at the outskirts of the Coma cluster, inside the cluster's turnaround radius.

The possibility that Coma cluster is perturbed was already suspected on the basis of optical measurements (Huchra et al. 1990; Colless \& Dunn 1996). Radio (Thierbach et al. 2003, and references therein) and X-ray measurements of ROSAT, ASCA and XMM-Newton (Briel et al. 1992; Honda et al. 1996; Briel et al. 2001; Neumann et al. 2003) confirmed this suspicion. The X-ray structure of the cluster detected by ROSAT and ASCA extends $\sim 1^{\circ}$ off the cluster core to the south-west

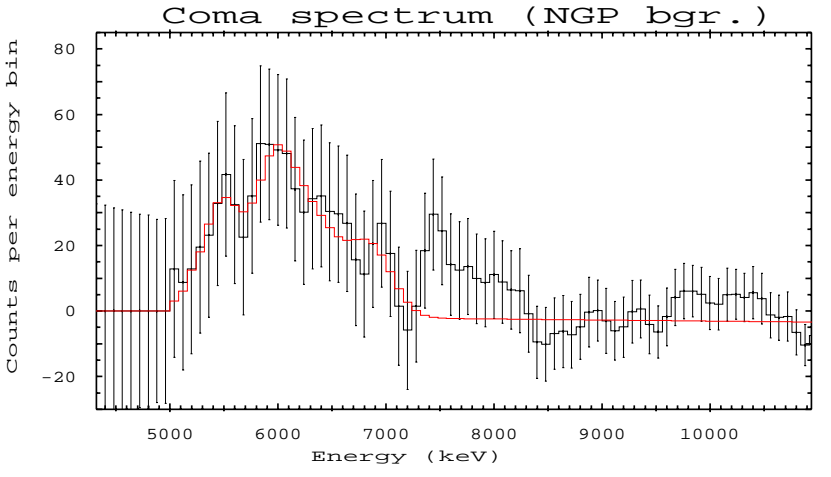

Fig. 7. Spectrum of the Coma $\gamma$-ray excess (excess 1). The solid line shows a fit to the excess emission.

direction (Briel et al. 1992; Honda et al. 1996). This structure, seen in the X-ray brightness and temperature distributions, is explained by the merging process of NGC 4839 with the Coma cluster.

The X-ray picture of the Coma cluster that is emerging after XMM-Newton observations (Briel et al. 2001; Neumann et al. 2003) is even more complicated. An additional filamentlike structure to the south-east of the cluster core was found with a much lower temperature of $\sim 1 \mathrm{keV}$ compared to the rest of the cluster that has $k T \geq 10 \mathrm{keV}$ (Neumann et al. 2003). As a possible scenario to explain this filament it is proposed that a small group of galaxies around two galaxies NGC 4921 and NGC 4911 is infalling into Coma gravitaional well with a non-zero impact parameter (Neumann et al. 2003). The $\gamma$-ray line excess 1 is also placed at the south-east outskirts of the cluster and thus supports this second merger scenario. We believe that in the Coma cluster a few of the galaxy groups, like [RPG97] 232 ( $Z=0.02286)$, and few Zwicky clusters with $\mathrm{RA} \sim 13 \mathrm{~h}$ and Dec $\leq 30^{\circ}$, are representatives of the filamentary structure that is moving from the south-east direction to merge with Coma.

The turnaround radius of the merging cluster can be calculated using the approach and notation of Ricker \& Sarazin (2001):

$R_{12} \approx 4.5\left(\frac{M_{1}+M_{2}}{10^{15} M_{\odot}}\right)^{1 / 3}\left(\frac{t_{\text {merge }}}{10^{10} \mathrm{yr}}\right)^{2 / 3} \mathrm{Mpc}$,

where $M_{1}$ and $M_{2}$ are the masses of the major cluster and of subcluster, respectively. For the mass of the Coma cluster of $1.8 \times 10^{15} \times h_{50}^{-1} M_{\odot}$ (Briel et al. 1992) this linear separation is of the order of $6 \mathrm{Mpc}$. This leads to an angular distance of the shock of $\sim 3.5^{\circ}$ from the Coma core at an early stage of the merger.

The merger shock with the linear scale $R$ of $\sim 1 \mathrm{Mpc}$ has a surface area of $\sim R^{2}$ and is expected to produce an energy input of the order of

$$
\begin{aligned}
\epsilon_{\text {input }} \sim & \frac{\rho v_{\mathrm{s}}^{2}}{R / v_{\mathrm{s}}} \\
\sim & 5 \times 10^{-28}\left(\frac{n}{10^{-3} \mathrm{~cm}^{-3}}\right)\left(\frac{v_{\mathrm{s}}}{1000 \mathrm{~km} \mathrm{~s}^{-1}}\right)^{3} \\
& \times\left(\frac{R}{1 \mathrm{Mpc}}\right)^{-1} \mathrm{erg} \mathrm{s}^{-1} \mathrm{~cm}^{-3}
\end{aligned}
$$


In simplified form the shock velocity $\left(v_{\mathrm{s}}\right)$ estimate can be derived from Eq. (31) given in Ricker \& Sarazin (2001). Assuming a shock front width of $\sim 10 \mathrm{kpc}$ one may expect to have an energy deposition in the Coma cluster merger region of the order of $\sim 1.2 \times 10^{45} \times h_{100}^{-2} \mathrm{erg} \mathrm{s}^{-1}$. This exceeds the $\gamma$-ray line luminosity of $\sim 2.0 \times 10^{44} \times h_{100}^{-2} \mathrm{erg} \mathrm{s}^{-1}$ detected by COMPTEL from the Coma cluster, but is much smaller than the X-ray luminosity of $L_{x}(0.01-40 \mathrm{keV}) \sim 8.8 \times 10^{45} \times$ $h_{100}^{-2} \mathrm{erg} \mathrm{s}^{-1}$ (Reiprich \& Böhringer 1999).

The angular distance of excess 1 of $\sim 3^{\circ}$ to Abell 1656 core is inside the angular turnaround radius, and is consistent with the merger at its early stage. This radius of the mass inflow in Coma is consistent also with the derived mass profile of the Coma, and corresponds to the caustics gradient at $\sim 6 \mathrm{Mpc} \mathrm{de}-$ rived from the galaxy distribution in the redshift diagram by Geller et al. (1999).

\subsection{Excess 2}

Excess 2 detected by COMPTEL has $\sim 5.3 \sigma$ significance for a 1 d.o.f. search. Its likelihood-ratio peaks between the positions of two massive galaxies in the Virgo cluster, M 87 and $\mathbf{M} 49$. These massive elliptical galaxies are thought to be gravitational centers of the northern Virgo subcluster (subcluster A) and of the southern subcluster (subcluster B), respectively (Binggelli et al. 1987, 1993). The redshift derived from the excess spectrum (Fig. 8) is $z=0.011 \pm 0.017$, which is not precise enough to identify potential counterparts of excess 2 , but is consistent with a Virgo origin. The $2 \sigma$ error box of this excess contains 4 Abell clusters, 7 Zwicky clusters, and 6 QSOs. The $\gamma$-ray excess position coincides with the maximum in the density distribution of the irregular and spiral galaxies of the Virgo cluster (Schindler et al. 1999), as well as with the region of enhanced temperature between M 87 and M 49 (Koyama et al. 1991; Ohashi et al. 1999; Kikuchi et al. 2000; Shibata et al. 2001).

Yasuda et al. (1997), using the Tully-Fisher relation for spiral galaxies, found evidence that the galaxy distribution in Virgo is largely elongated along the line of sight from 12 to $30 \mathrm{Mpc}$. This may imply that if multiple mergers happen in Virgo they may be ongoing at different redshifts and with different radially projected bulk velocities. Such variety will eventually lead to the broadened, flat topped $\gamma$-ray lines. The spectrum of the $\gamma$-ray excess from this region in Virgo indeed contains a broadened line of ${ }^{16} \mathrm{O}$ at $\sim 6.1$ (Fig. 8), consistent with the hypothesis of multiple mergers at different redshifts. The enhanced temperature region in Virgo found by ASCA was explained by the inflow of groups of galaxies (Ohashi et al. 1999; Kikuchi et al. 2000; Shibata et al. 2001) towards the cluster gravitational center. The quite narrow merger shock of M 49 discussed by Irwin \& Sarazin (1996) is placed $\sim 4$ arcmin to the north of M 49, while the enhanced temperature region between $\mathrm{M} 49$ and M 87 found by ASCA is much larger. It spreads from $\sim 8^{\circ}$ to $11^{\circ}$ in declination, along the north-south scan of the Virgo temperature distribution (Shibata et al. 2001). This region with an area of $\sim 2.8 \mathrm{deg}^{2}$ has a $\sim 4$ times higher $\mathrm{X}$-ray emissivity than the region with radius

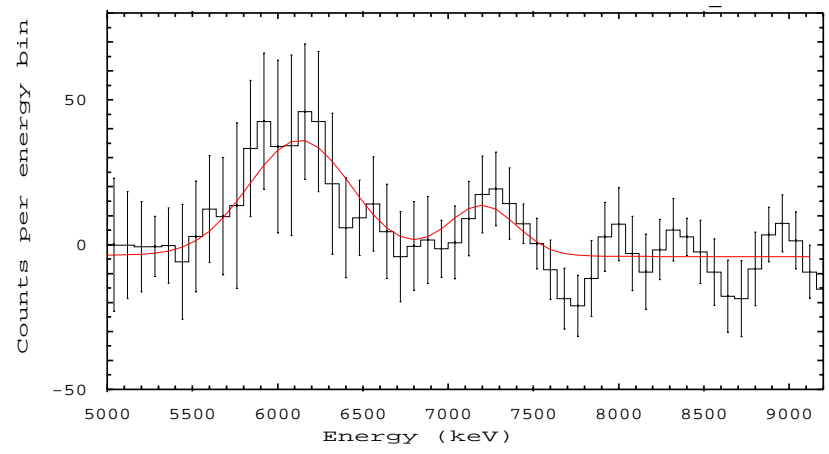

Fig. 8. Spectrum of the Virgo $\gamma$-ray excess (excess 2) at a position between M 87 and M 49. The solid line shows a fit to the excess emission.

of $1^{\circ}$ around $\mathrm{M} 49$. The total luminosity from this strip is of the order of $L_{x}(2.0-10.0 \mathrm{keV}) \sim 4.0 \times 10^{44} \mathrm{erg} \mathrm{s}^{-1}$ (Shibata et al. 2001).

The temperature enhancement is resolved into four subpeaks with typical sizes of $\sim 300 \mathrm{kpc}$, that allows Shibata et al. (2001) to claim a multiple merger of the galaxy groups. Possible infalling groups can be represented by the Abell 1541C cluster, and/or by the three galaxy groups RSCG 57 and RSCG 60 at $z \approx 0.0020$, and [M98j] 174 at $z \approx 0.00545$, under the plausible assumption that galaxy groups merging into Virgo have to be at a redshift equal or close to that of the Virgo cluster. We note that one peak in the scan A is likely consistent with Abell 1553. The suggested potential candidates seem to strenghten the case of multiple mergers of galaxy groups with the Virgo cluster. With this hypothesis in mind we estimate the energetics of such multiple merger event and the plausibility for its detection in $\gamma$-ray lines.

Preliminary estimates, based on the $\gamma$-ray flux and distance to the Virgo cluster (taken to be $20 \mathrm{Mpc}$ ), lead to the conclusion that for the measured $\gamma$-ray line flux $\left(F_{16 \mathrm{O}}=(1.12 \pm\right.$ $0.27) \times 10^{-5}$ photons $\left.\mathrm{cm}^{-2} \mathrm{~s}^{-1}\right)$ and for any reasonable time scale of a merger event the total energy necessary to produce the observed $\gamma$-ray line emission has to have a value of up to $2 \times 10^{63}$ ergs. It is difficult to account for such an energy by a process other than a merger. For example, to explain the cluster thermal energy input by SNe one would need $\sim 10^{12} \mathrm{SNe}$ to heat the gas, and such an input will lead to an observable overproduction of iron, which is excluded by observations (Finoguenov et al. 2001). Alternatively, an energy input from 10 quasars with an output of $\sim 10^{46} \mathrm{ergs} \mathrm{s}^{-1}$ for $\sim 10^{9} \mathrm{yr}$, can produce a similar effect, but no quasars of this high luminosity positionally consistent with the error box of excess 2 were detected in $\gamma$-rays neither by EGRET (Hartman et al. 1999) nor by COMPTEL (Collmar et al. 2001).

We can evaluate the energy input in such a merger using Eq. (4). In the case of Virgo the linear size of the merger shock front can be derived as $\approx 300 \mathrm{kpc}$ from the ASCA temperature profile (Shibata et al. 2001). The only parameter which is uncertain, is the shock velocity $v_{\mathrm{s}}$, which depends on the mass of the major accretor. If we take the total mass of the Virgo cluster $\sim 1.3 \times 10^{15} M_{\odot}$ (Tully \& Shaya 1984 ; Tully \& Shaya 1998) as the accretor mass, we will have an energy deposition at the 
rate of $4.15 \times 10^{44} \mathrm{erg} \mathrm{s}^{-1}$ for the single galaxy group merger, with the group mass of $10^{13} M_{\odot}$. In the case of 4 galaxy groups merger, where number 4 is taken from the number of temperature peaks of ASCA's scan B (Shibata et al. 2001), we will have an energy deposition of the order of $1.7 \times 10^{45} \mathrm{erg} \mathrm{s}^{-1}$. This energy deposition exceeds the sum of the $\gamma$-ray luminosity $\left(\sim 6.24 \times 10^{42} \times h_{100}^{-2} \mathrm{erg} \mathrm{s}^{-1}\right)$ and of the X-ray luminosity $\left(\sim 4.0 \times 10^{44} \times h_{100}^{-2} \mathrm{erg} \mathrm{s}^{-1}\right)$ from the region of the enhanced temperature in Virgo by a factor of 4 .

Alternatively, by taking a Virgo cluster mass of $1.5 \times$ $10^{14} M_{\odot}$ to $6.0 \times 10^{14} M_{\odot}$, as derived by Böhringer et al. (1994) under the assumption that the cluster is relaxed, and by taking the merger shock velocity as the $3 \mathrm{D}$ velocity dispersion of the galaxies in Virgo which is $\sim 1200 \mathrm{~km} \mathrm{~s}^{-1}$ (Binggeli et al. 1993) one can get an energy deposition rate estimate of $\sim 2.0 \times$ $10^{44} \mathrm{erg} \mathrm{s}^{-1}$ for the multiple merger scenario. We consider this value as a lower limit to an energy deposition rate, which still exceeds the $\gamma$-ray luminosity detected by COMPTEL. In reality one may have a more complex scenario involving a filamentary structure merger with the main Virgo cluster. We reiterate here that the Virgo cluster extends from 12 to $30 \mathrm{Mpc}$ along the line of sight (Yasuda et al. 1997). We conclude that the above energetics estimate makes a scenario of a multiple galaxy group merger with the main body of the Virgo cluster plausible, but this conclusion is highly dependent on the $3 \mathrm{D}$ geometry of the cluster (Binggelli et al. 1987, 1993; Yasuda et al. 1997; Schindler et al. 1999). Better $\gamma$-ray line position measurement, and confirming measurements by XMM-Newton of the X-ray gas temperature enhancement (structure) in this region of Virgo will help to interpret the multiple merger scenario in Virgo cluster.

\section{Discussion and summary}

We have presented detections of the nearby, rich Coma and Virgo galaxy clusters via their $\gamma$-ray line emission from ${ }^{16} \mathrm{O}$, excited by subrelativistic protons accelerated from the thermal pool in a merger event. What kind of arguments support the proposed interpretation?

During a merger event, shocks are driven into the intracluster medium, that dissipate energies of up to $\sim 3 \times 10^{63}$ ergs. Particles, electrons and ions, will be accelerated in shocks even if merger shocks are having small Mach numbers $(M)$, typically of 2 (Gabici \& Blasi 2003; Berrington \& Dermer 2003).

Protons with a kinetic energy as low as $\sim 10 \mathrm{MeV}$ can produce ${ }^{16} \mathrm{O}$ excitation $\gamma$-ray line emission (Ramaty et al. 1996) in interactions with the ICM as a target. Such protons will have a Coulomb cooling time of $\tau_{\text {ion }} \approx 3 \times 10^{7}$ yr in the ICM with a density $\sim 10^{-3} \mathrm{~cm}^{-3}$. Protons with energies of $\sim 100 \mathrm{MeV}$ have Coulomb cooling times of $2.7 \times 10^{8} \leq \tau_{\text {ion }} \leq 8.7 \times 10^{9} \mathrm{yr}$, depending on the ionization state of the ICM gas and the same density of $10^{-3} \mathrm{~cm}^{-3}$. This provides a lower limit for $\tau_{\text {ion }}$ for the fully ionized plasma.

A shock with a compression factor $r\left(=u_{1} / u_{2}\right)$ and a Mach number $\mathcal{M}\left(=v_{\mathrm{s}} / v_{\text {sound }}\right)$ can accelerate particles to a power law in momentum $f(p) \propto p^{-\alpha}$, where $\alpha$ is related to the Mach number and compression factor by the following expression:

$\alpha=\frac{r+2}{r-1}=2 \frac{\mathcal{M}^{2}+1}{\mathcal{M}^{2}-1}, \quad(\approx 3.3$ for $M=2)$.

The acceleration occurs diffusively, where particles are crossing back and forth the shock front, gaining at each crossing and recrossing an amount of energy proportional to the energy of the particle. The maximum proton energy in such an acceleration is the result of a balance between the acceleration rate and either the energy loss rate or the rate of escape from the acceleration region. The acceleration time, as a function of the particle energy $E$, is defined by the expression (Gabici \& Blasi 2003)

$\tau_{\mathrm{acc}}(E)=\frac{3}{u_{1}-u_{2}} D(E)\left[\frac{1}{u_{1}}+\frac{1}{u_{2}}\right]=\frac{3 D(E)}{v^{2}} \frac{r(r+1)}{r-1}$,

where $D(E)$ is a diffusion coefficient of a particle with energy $E$. For the form of $D(E)$ proposed by Blasi \& Colafrancesco (1999) the acceleration time can be defined as follows:

$\tau_{\mathrm{acc}}(E) \approx 6.9 \times 10^{13} B_{\mu}^{-1 / 3} L_{20}^{2 / 3} E(\mathrm{GeV})^{1 / 3} v_{8}^{-2} g(r) \quad \mathrm{s}$,

where $v_{8}=\frac{v}{10^{8} \mathrm{~cm} / \mathrm{s}}, g(r)=r(r+1) /(r-1), v=u_{1}, B_{\mu}$ is the magnetic field in microgauss, $L_{20}$ is the largest scale in the magnetic field power spectrum in units of $20 \mathrm{kpc}$, and $r=u_{1} / u_{2}$ (see Gabici \& Blasi 2003).

For protons with $E_{\mathrm{p}} \leq 100 \mathrm{MeV} \tau_{\text {acc }}$ has a value of $\sim 1.5 \times$ $10^{7} \mathrm{yr}$ for $v_{8} \sim 2.0$ and $B_{\mu} \sim 0.01 \mu \mathrm{G}$. For the value of $B_{\mu} \sim$ $1 \mu \mathrm{G}$, which is implied by the radio measurements of the Coma $C$ radio spectrum (Thierbach et al. 2003), the value of $\tau_{\text {acc }}$ will be even smaller, of $\sim 3.3 \times 10^{6} \mathrm{yr}$.

The model that considers the generation of $\gamma$-ray line emission in galaxy clusters by subrelativistic nuclei was suggested by Dogiel (2001). This model explains the hard X-ray spectrum of Coma detected by PDS on board of Beppo-SAX (Fusco-Femiano et al. 1999), by energy losses of the subrelativistic CRs in the cluster halo interacting with the background electrons and nuclei of ICM. The spectrum of the subrelativistic protons was considered to be a power law with an exponential cut-off, which is produced either by proton injection in the center of the cluster, or by protons that are accelerated in situ (Dogiel 2001). The latter seems to be energetically more efficient (Dogiel 2001) and attractive in view of the radio measurements confirming to the idea of in-situ acceleration (Thierbach et al. 2003).

The X-ray continuum in the model of Dogiel (2001) is produced at $E_{x}<\left(m_{\mathrm{e}} / M_{\text {ion }}\right) E_{\max } \mathrm{keV}$ by thermal bremsstrahlung of background electrons, and by inverse bremsstrahlung of subrelativistic protons with kinetic energy $E_{\mathrm{p}} \leq E_{\max }$, or by ions with $E_{\text {ion }} \leq\left(E_{\max } / n\right)$, where $E_{\max }$ is approximately equal to an exponential cut-off energy of CR spectrum. There is also a contribution to the $\mathrm{X}$-ray continuum from $\mathrm{CR}$ interaction with ICM gas through molecular-orbital (MO) and radiative electron capture (REC) on CR nuclei (Dogiel et al. 1998). Such direct electron captures, like MO or REC, are important in cases with high enrichment of CRs by heavy ions. For light 
ions (hydrogen, helium) multistep knock-on (KO) electrons are more important in producing X-rays (Chu et al. 1981; Dogiel et al. 1998; Tatischeff et al. 1998). Bremsstrahlung of KO electrons is used to explain an X-ray continuum at $\left(m_{\mathrm{e}} / M_{\text {ion }}\right) E_{\max }<$ $E_{x}<4\left(m_{\mathrm{e}} / M_{\text {ion }}\right) E_{\max } \mathrm{keV}$ (Dogiel 2001). Since in the model of Dogiel (2001) the hard X-ray and $\gamma$-ray fluxes are produced by the same subrelativistic nuclei interacting with the intracluster gas (intergalactic matter), their ratio is independent of the gas density. For the power-law spectrum of the subrelativistic nuclei this ratio is also independent of the density of the subrelativistic CRs. One may use the X-ray flux of Coma at energies where the non-thermal component is dominant, for example at $E_{x}=70 \mathrm{keV}$ (see PDS spectrum by Fusco-Femiano et al. $1999)$, where $F_{x}(70 \mathrm{keV})=2.7 \times 10^{-6} \mathrm{ph} \mathrm{cm}^{-2} \mathrm{~s}^{-1} \mathrm{keV}^{-1}$, to calculate the expected intensity of the oxygen line(s), for the photon index of subrelativistic CRs of $\sim 1.0$ (Dogiel 2001), as $F_{16} \mathrm{O}=1.6 \times 10^{-5} \mathrm{ph} \mathrm{cm}^{-2} \mathrm{~s}^{-1}$. The line flux from Coma detected by COMPTEL has a value of $F_{16} \mathrm{O} \sim 1.4 \times$ $10^{-5} \mathrm{ph} \mathrm{cm}^{-2} \mathrm{~s}^{-1}$, which is very close to the value calculated above.

We note, though, that in the early phases of a merger, for a time lapse of $\leq \tau_{\text {loss }}$, a much steeper proton spectrum with a photon index of 3 to 5 is more likely (Berrington \& Dermer 2003). A steep spectrum is produced because of the low Mach numbers at the cluster periphery, where shock starts to develop plunging into the ICM with the density of $\sim 3 \times 10^{-4} \mathrm{~cm}^{-3}$. Inside the denser cluster core the power law index will decrease approaching a value of $\sim 2.2$ with the shock advancing towards the cluster core (Berrington \& Dermer 2003). Note that the power law index of the detected hard non-thermal X-ray component of the Coma core region is $\sim 1.6$, with a $90 \%$ confidence range of 0.7-2.5 (Fusco-Femiano et al. 1999), or $2.1 \pm$ 0.5 , with a $90 \%$ confidence (Rephaeli \& Gruber 2002), both estimates being consistent with the shock in the region of the cluster core.

To produce the $\gamma$-ray flux detected by COMPTEL from Coma, one needs an energy deposition by subrelativistic protons of the order of $L_{\mathrm{p}} \sim 8.0 \times 10^{48} \mathrm{erg} \mathrm{s}^{-1}$, for the uncertain efficiency of the $\gamma$-ray production by protons of $\eta \sim 10^{-5}$, as assumed by Dogiel et al. (1997). The same energetics of $\sim(3-8) \times 10^{48} \mathrm{erg} \mathrm{s}^{-1}$ is needed to produce the observed hard $\mathrm{X}$-ray emission of Coma (Dogiel 2001). We note that the above comparison of the COMPTEL measured ${ }^{16} \mathrm{O} \gamma$-ray line flux with that predicted by the model of Dogiel (2001) is based on the hard X-ray measurement performed for the Coma cluster core region with the size of $\sim 1^{\circ}$ (Fusco-Femiano et al. 1999). Therefore regions of hard X-ray emission and of $\gamma$-ray line emission do not overlap. It is clear that consistency of the measured and of the model predicted $\gamma$-ray line flux is fortuitous, but the above comparison is useful to get an estimate of the parameter ranges allowed in such a model.

To identify the subclusters responsible for the COMPTEL line excesses in Coma and Virgo one will need a better localisation ( $\sim 1$ arcmin) of the corresponding soft $\gamma$-ray (hard X-ray) excess and a better measurements of the line energy. Both imaging (IBIS) and spectroscopical (SPI) instruments of the ESA space mission INTEGRAL, launched in October 2002, have the abilities to solve these tasks. ICM abundance measurements for the $\gamma$-ray line excess region that can be provided by XMM-Newton will help to enhance our understanding and the interpretation of the results discussed above.

Before this work, the existence of a non-thermal population of CRs in galaxy clusters was supported by the detections of hard X-rays from Coma and A2319 (Fusco-Femiano et al. 1999; Rephaeli et al. 1999; Gruber \& Rephaely 2002; Rephaeli $\&$ Gruber 2002), and also from presumably merging rich cluster Abell 3667 (Mazzota et al. 2002), which contains the largest known diffuse radio source (Röttgering et al. 1997). The radio spectrum of Coma C (Thierbach et al. 2003, and references therein) also points to the presence of in situ accelerated relativistic electrons in Coma. Contrary to these X-ray and radio measurements, the detection of excitation $\gamma$-ray lines from clusters Coma and Virgo by COMPTEL provide the first direct evidence for the non-thermal nucleonic CR component generation in the merger event(s).

To summarize we note that the presented method and results of the galaxy clusters study via their emission in the excitation $\gamma$-ray lines has the potential to become an important tool in probing the dynamics of the merging clusters. Indeed, the $\gamma$-ray line flux and the spectrum provide rather direct means to evaluate the energy input into the ICM in an ongoing merger, and the potential to reconstruct the 3D geometry of the merger. By applying this tool at much better sensitivity one can gain information on the spectrum and maximum energy of protons produced by the merger shock, i.e., on the energy density of non-thermal particles in the cluster.

The hot spots of $\gamma$-ray line emission detected by COMPTEL will be useful for planning of INTEGRAL and of XMM-Newton observations. Such observations will confirm and hopefully extend the number of confirmed merging galaxy clusters, as well as providing key information on the non-thermal component energy density in the evolving large scale structures.

Acknowledgements. We acknowledge comments to this work by Dr. V. Schönfelder and discussions with S. Schindler, R. Staubert and H. Völk. We are grateful to Dr. V. Tatischeff for pointing to us (AI) the nuclear deexcitation $\gamma$-ray line code developed by R. Ramaty, R. Murphy and B. Kozlovsky (see http://lheawww.gsfc.nasa.gov/users/ramaty/ViewPubs/ ramaty.html), which we have used to produce spectra of Fig. 1. This research has made use of the NASA/IPAC Extragalactic Database (NED) which is operated by the Jet Propulsion Laboratory, California Institute of Technology, under contract with the National Aeronautics and Space Administration, as well as of NASA's Astrophysics Data System Service.

\section{References}

Berrington, R. C., \& Dermer, C. D. 2003, ApJ, 594, 709

Binggeli, B., Tammann, G. A., \& Sandage, A. 1987, AJ, 94, 251

Binggeli, B., Popescu, C. C., \& Tammann, G. A. 1993, A\&AS, 98, 275

Blasi, P., \& Colafrancesco, S. 1999, Astropart. Phys., 15, 223

Bloemen, H., Hermsen, W., Swanenburg, B. N., et al. 1994, ApJS, 92, 419

Bloemen, H., Morris, D., Knödlseder, J., et al. 1999, ApJ, 521, L137

Bloemen, H., Bennett, K., Collmar, W., et al. 2000, AIP CP-510, 586 
Böhringer, H., \& Morfill, G. 1988, ApJ, 330, 609

Böhringer, H., Briel, U., Schwarz, R., et al. 1994, Nature, 368, 828

Briel, U. G., Henry, J. P., \& Böhringer, H. 1992, A\&A, 259, L31

Briel, U. G., Henry, J. P., Lumb, D. H., et al. 2001, A\&A, 365, L60

Chu, T. C., Ishii, K., Yamadera, A., Sebata, M., \& Norita, S. 1981, Phys. Rev. A, 24, 1720

Colafrancesco, S. 2002, A\&A, 396, 31

Colless, M., \& Dunn, A. M. 1996, ApJ, 458, 435

Collmar, W., Schönfelder, V., Bloemen, H., et al. 2001, AIP CP-587, 271

Dogiel, V. A., Freyberg, M. J., Morfill, G. E., \& Schönfelder, V. 1997, AIP CP-410, 1069

Dogiel, V. A., Ichimura, A., Inoue, H., \& Masai, K. 1998, PASJ, 50, 575

Dogiel, V. 2001, ESA SP-459, 139

Ebeling, H., Edge, A. C., Böhringer, H., et al. 1998, MNRAS, 301, 881

Fang, T., Sembach, K. R., \& Canizares, C. R. 2003, ApJ, 586, L49

Finoguenov, A., Reiprich, T. H., \& Böhringer, H. 2001, A\&A, 368, 749

Fusco-Femiano, R., Dal Fiume, D., Feretti, L., et al. 1999, ApJ, 513, L21

Fusco-Femiano, R., Dal Fiume, D., De Grandi, S., et al. 2000, ApJ, 534, L7

Gabici, S., \& Blasi, P. 2003, ApJ, 583, 695

Geller, M. J., Diaferio, A., \& Kurtz, M. J. 1999, ApJ, 517, L23

Gruber, D., \& Rephaeli, Y. 2002, ApJ, 565, 877

Hartman, R. C., Bertsch, D. L., Bloom, S. D., et al. 1999, ApJS, 123, 79

Honda, H., Hirayama, M., Watanabe, M., et al. 1996, ApJ, 473, L71

Huchra, J. P., Geller, M. J., de Lapparent, V., Corwin, H. G., Jr. 1990, ApJS, 72, 433

Irwin, J. A., \& Sarazin, C. L. 1996, ApJ, 471, 683

Iyudin, A. F., Diehl, R., Bloemen, H., et al. 1994, A\&A, 284, L1

Iyudin, A. F., Bennett, K., Bloemen, H., et al. 1995, A\&A, 300, 422

Iyudin, A. F., Bennett, K., Bloemen, H., et al. 1996, A\&A, 311, L21

Iyudin, A. F., Schönfelder, V., Bennett, K., et al. 1998, Nature, 396, 142

Kaastra, J. S., Lieu, R., Tamura, T., et al. 2003, A\&A, 397, 445

Kawasaki, W., \& Totani, T. 2002, ApJ, 576, 679

Kikuchi, K., Itoh, C., Kushino, A., et al. 2000, ApJ, 531, L95

Koyama, K., Takano, S., \& Tawara, Y. 1991, Nature, 350, 135

Kozlovsky, B., Murphy, R. J., \& Ramaty, R. 2002, ApJS, 141, 523
Loeb, A. 2002, New Astr., 7, 279

Mazzota, P., Fusco-Femiano, R., \& Vikhlinin, A. 2002, ApJ, 569, L31

Miniati, F., Ryu, D., Kang, H., \& Jones, T. W. 2001, ApJ, 559, 59

Neumann, D. M., Lumb, D. H., Pratt, G. W., \& Briel, U. G. 2003, A\&A, 400, 811

Nicastro, F., Zezas, A., Drake, J., et al. 2002, ApJ, 573, 157

Norman, M. L., \& Bryan, G. L. 1999, LNP, 530, 106

Ohashi, T., Kikuchi, K., Yamasaki, N. Y., \& Shibata, R. 1999, MPE-Report, 271, 143

Paerels, F., Rasmussen, A., Kahn, S., et al. 2002, MPE Report, 281, 57

Ramaty, R., Kozlovsky, B., \& Lingenfelter, R. E. 1996, ApJ, 456, 525

Reimer, O., Pohl, M., Sreekumar, P., \& Mattox, J. R. 2003, ApJ, 588, 155

Reiprich, T. H., \& Böhringer, H. 1999, ApJ, 320, 296

Rephaeli, Y., Gruber, D. E., \& Blanco, P. 1999, ApJ, 511, L21

Rephaeli, Y., \& Gruber, D. 2002, ApJ, 579, 587

Ricker, P. M., \& Sarazin, C. L. 2001, ApJ, 561, 621

Ritchie, B. W., \& Thomas, P. A. 2002, MNRAS, 329, 675

Röttgering, H. J. A., Wieringa, M. H., Hunstead, R. W., \& Ekers, R. D. 1997, MNRAS, 290, 577

Sarazin, C. L. 2002, in Merging Processes in Clusters of Galaxies (Dordrecht: Kluwer), ASSL, 272, 1

Savage, B. D., Sembach, K. R., Wakker, B. P., et al. 2003, ApJS, 146, 125

Scharf, G. A., \& Mukherjee, R. 2002, ApJ, 580, 154

Schindler, S., Binggeli, B., \& Böhringer, H. 1999, A\&A, 343, 420

Schönfelder, V., Aarts, H., Bennet, K., et al. 1993, ApJS, 86, 657

Schönfelder, V., Bennet, K., Blom, J. J., et al. 2000, A\&AS, 143, 145

Shibata, R., Matsushita, K., Yamasaki, N. Y., et al. 2001, ApJ, 549, 228

Sreekumar, P., Bertch, D. L., Dingus, B. L., et al. 1996, ApJ, 464, 628

Tatischeff, V., Ramaty, R., \& Kozlovsky, B. 1998, ApJ, 504, 874

Thierbach, M., Klein, U., \& Wielebinski, R. 2003, A\&A, 397, 53

Tully, R. B., \& Shaya, E. J. 1984, ApJ, 281, 31

Tully, R. B., \& Shaya, E. J. 1998, Evolution of Large-Scale Structure, Proc. of the MPA-ESO Cosmology Conf., ed. A. J. Banday, R. K. Sheth, \& L. N. Da Costa, 296

van Dijk, R. 1996, Ph.D. Thesis, Amsterdam Univ.

Weidenspointner, G. 1999, Ph.D. Thesis, TU München

Yasuda, N., Fukugita, M., \& Okamura, S. 1997, ApJS, 108, 417

Zsargo, J., Sembach, K. R., Howk, J. C., \& Savage, B. D. 2003, ApJ, 586,1019 